\title{
Route Planning of UAV Based on Improved Ant Colony Algorithm
}

\author{
Zhengxiang Qian * \\ New Star Research Inst. of Applied Tech. in Hefei \\ 451, Huangshan Road, Hefei \\ Hefei, China \\ E-mail: qianzhengxiang@ sina.com \\ * Corresponding Author \\ Guocheng Wang \\ New Star Research Inst. of Applied Tech. in Hefei \\ 451, Huangshan Road, Hefei \\ Hefei, China
}

\author{
Jingen Wang \\ New Star Research Inst. of Applied Tech. in Hefei \\ 451, Huangshan Road, Hefei \\ Hefei, China \\ Yongxin Shi \\ New Star Research Inst. of Applied Tech. in Hefei \\ 451, Huangshan Road, Hefei \\ Hefei, China
}

\begin{abstract}
On the basis of analyzing the tactical characteristics and the mission requirements of UAV, a route planning model is established, which is composed of a comprehensive threat model, a performance constraint model and a mission effectiveness model. A dual population genetic ant colony algorithm is designed, with which dual population ant colony can be searched and iterated independently at same time. In the iterative process, bidirectional dynamic adjust adaptively the volatile coefficient of the pheromone which is limited within a certain range. This algorithm can avoid local optimum and stagnation in the search and iteration. Finally The improved ant colony algorithm is applied to the route planning of $\mathrm{UAV}$, and the feasibility and effectiveness of this method is verified by simulation.
\end{abstract}

Keywords- UAV; Ant Colony Algorithm; Route Planning; Dual population; GA Algorithm

\section{INTRODUCTION}

In recent years, the UAV reconnaissance has become one of the important means of battlefield intelligence with Unmanned Aerial Vehicle Route Planning a key technique in mission planning system. The purpose of this method is to find the optimal or a better flight Route in possible time allowed in reality. It is the technical guarantee to ensure the improvement of Unmanned Aerial Vehicles for the efficiency of the aircraft and successful completion of assigned tasks. ${ }^{[1][2]}$ Ant colony algorithm is a new intelligent algorithm which enjoys universality and robustness and can be easily combined with other methods. It has now become a hot research topic in the field of artificial intelligence and bionic optimization. Ant colony algorithm can bring about route planning for unmanned combat aircraft and obtain the solution of the optimal route. Many scholars at home and abroad have studied the route planning method based on ant colony algorithm.

\section{ROUTE PlanNING OF UAV}

\section{A Key Technology of UAV's Route Planning}

Air route planning involves multiple disciplines and specialties such as flight mechanics, image processing, navigation, operational effectiveness analysis, radar, fire control, artificial intelligence, computers and automatic control and so on. ${ }^{[3]}$ This subject is thus comprehensive and difficult with key problems shown in Fig. 1:

\begin{tabular}{|c|c|c|c|}
\hline $\begin{array}{l}\text { Battlefield information } \\
\text { acquisition and processing }\end{array}$ & $\begin{array}{l}\text { building reconnaissance } \\
\text { task mode }\end{array}$ & $\begin{array}{l}\text { Deploying trajectory } \\
\text { planning algorithm }\end{array}$ & $\begin{array}{l}\text { Tracking and controlling } \\
\text { flight trajectory }\end{array}$ \\
\hline
\end{tabular}

Figure 1. Key technology of UAV's Route Planning

The terrain information and the situation which route planning needs is the most important and can generally be obtained by satellite and other methods..

The obtained information processed as the basis for the building of UAV's threat model and the degree of model application complexity affects the route optimization precision and the speed of the algorithm.

Configured with the appropriate algorithm is the soul of route planning. Ant colony algorithm is a new intelligent algorithm which enjoys versatility and robustness and can be easily combined with other methods.
It has now become a hot research topic in the field of bionic optimization and artificial intelligence.

The UAV Trajectory controller is designed according to the planning trajectory and the guidance law, which will make the aircraft fly along the route.

\section{B The Domestic Research Status and Shortages}

Through the analysis of domestic documentation about UAV route planning, it is found that most UAV route planning is of theoretical analysis and most research work is carried out for the reduction of the complexity of the 
algorithm structure. Most traditional planning methods are based on a predetermined cost function to generate a route with minimum cost. In many applications, however, the resulting minimum cost route cannot meet the actual requirements. To apply these methods in UAV route planning, two problems must be solved: (1)Now most of the studies are mainly theoretical with less research for the application of the methods. The constraint conditions are too simple to really reflect UAV performance in a task. (2)Though route planning algorithm is varied with a lot of improvement in many documentation materials, more suitable algorithm is needed for more complex route planning models. ${ }^{[4][5]}$

Therefore, a deeply study based on existing route planning methods is needed to meet the operational requirements of a certain UAV route planning, especially to solve the problems like how to build a more close-toactual-combat environment model and cost function and route search algorithm with more superior configuration.

\section{DuAl Populations Genetic Ant COLONY ALGORITHM}

Ant colony algorithm is a new bionic algorithm derived from the biological world in the nature. In the short time of more than ten years since its birth, it enjoys fast development and obvious advantages like strong robustness, distributed computing, easy parallel implementation, etc. Yet the basic ant colony algorithm has defects, 1 the algorithm generally needs longer search time, the complexity of the ant colony algorithm can reflect this; 2 the ant colony algorithm is prone to stagnation phenomenon in the process of constructing solution and positive feedback mechanism aiming at strengthening a solution of better performance. Namely all individuals have found the solutions identical with the search carried out to certain extent instead of furthering the research in solution space or finding a better solution.

Therefore, in this paper, the basic ant colony algorithm is improved as stated in the following four aspects:

(1) Firstly, using genetic algorithm for solution through basic operations like coding, individual evaluation, selection, crossover and mutation genetic algorithm so as to find some of the better routes in the planning space. Then initial pheromone of ant colony algorithm is formed through the route according to certain transformation rules.

(2) Improving single population search iteration in the basic ant colony algorithm for independent and synchronous dual population search iteration. Results are compared after two different populations respectively searching for solution at the same time. When iteration algorithm falls into local optimum, pheromone corresponding to the paths of different populations is exchanged. The accumulation of pheromone situation is not identical because of different initial parameters and operation of different populations. Information exchange of different populations can break the original ant colony search state and further expand the search space so as to break through the limitation of local optimum, thus get closer to global optimal solution.

(3) Referring to adaptive ant colony algorithm, improvement strategy of two-way adaptive adjusting pheromone volatilization coefficient is applied to achieve the purpose of further expanding of the search space and make the search results more advantageous to converge to global optimal solution.

(4)Referring to the smallest and largest ant systems, the pheromone of different population trails are respectively restricted within $\tau_{\min }-\tau_{\max }$ to avoid local optimal solution for the algorithm because of the high pheromone in certain paths.

This method can guarantee good initial pheromone distribution for the ant colony while expending the search space to avoid local optimum in solving process.

\section{ROUTE PlanNING OF UAV BASED ON IMPROVED ANT COLONY ALGORITHM}

\section{A. Grid dividing of route planning space}

The battlefield environment of a certain type of UAV in operation is shown in figure 2. Within a square battlefield area of $200 \mathrm{~km}$ in length and width, a UAV launch and collection point for $A$, four targets for $M_{1}, M_{2}$, $M_{3}, M_{4}$. To build $X O Y$ coordinate system by the edges of the battlefield area for $X$ axis and $Y$ axis, and then equal $X$ axis and $Y$ axis on the battlefield, get the grid as Fig. 2.

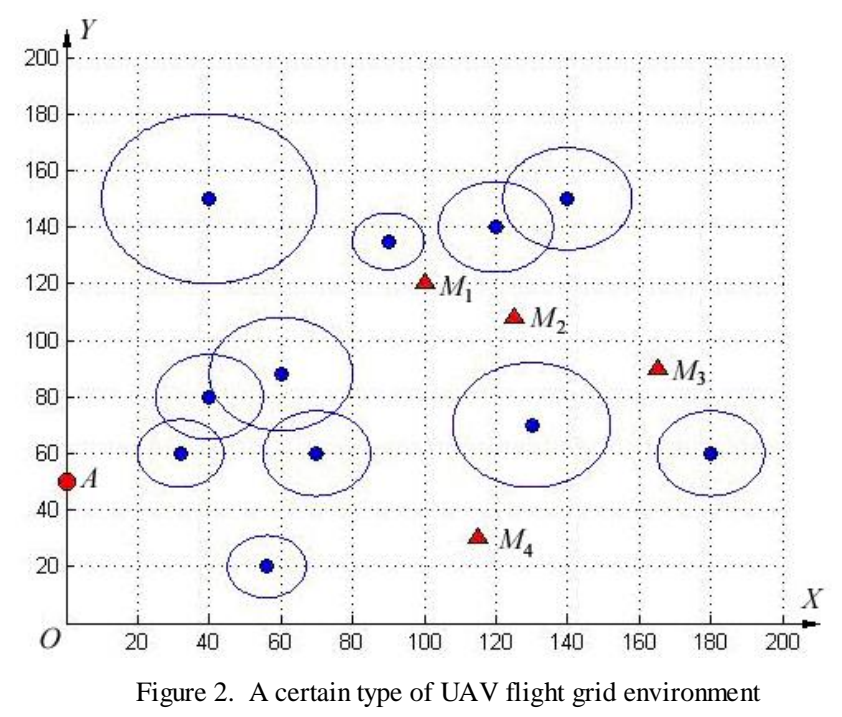

\section{B. Route planning model}

\section{1) Route planning comprehensive threat model:}

The threat source of UAV reconnaissance mainly includes radar, missile, air defense weapons like antiaircraft guns and the factors that affect flight safety such as towering mountain peaks, bad weather and others. ${ }^{[6]}$ They are all weighted items that the threat level is calculated; Modeling all threat sources respectively to get the following theoretical threat cost function after each item ${ }^{[7]}$ is weighted, the threat source model becomes a part of the reconnaissance evaluation model.

$$
W_{t}=\tau_{1} \cdot P_{S f}+\tau_{2} \cdot P_{R d}+\tau_{3} \cdot P_{M d}+\tau_{4} \cdot P_{M g}+\tau_{5} \cdot P_{W e}
$$

Among them, $W_{t}$ stands for the threat cost, $P_{S f}$ stands for the probability of terrain threat to aircraft, $P_{R d}$ stands for the probability of the aircraft discovered by radar, $P_{M d}$ stands for the probability of the aircraft threatened by air missile , $P_{M g}$ stands for the probability of the aircraft 
threatened by anti-aircraft gun, $P_{W e}$ stands for the probability of the aircraft threatened by bad weather, $\tau_{i}(i$ $=1,2,3,4,5)$ stands for the weighted coefficient of each item.

2) UAV's performance constraint model: ${ }^{\text {[8] [9] }}$

Researchers assume that UAV flies in the same altitude at a constant speed, performance constraint mainly considers fuel cost, namely, maximum range price. This limits the length of the route which must be less than or equal to a preset maximum distance. It depends on the fuel carried by aircraft and the flight time allowed for the aircraft. Under the assumed conditions as mentioned above, fuel consumption cost is proportional to the flying route length $\mathrm{L}$, so either of the two needs to be considered to represent all constraints. Set the maximum air route length $L_{\max }$, the constraint can be written as:

$$
\sum_{i} l_{i} \leq l_{\max }
$$

\section{3) UAV's mission decision-making influence model:}

When the UAV performs reconnaissance tasks, commander's expected goal is to find targets effectively in the limited flight time and range at a minimum price. It is often a trade-off between the two. Commanders need to make a decision.

\section{Set algorithm elements}

\section{1) The performance indexes of route planning:}

A certain type of UAV's route planning performance index is expressed as:

$$
W=k_{w} a W_{t}+\left(1-k_{w}\right) W_{x} \quad\left(0 \leq k_{w} \leq 1\right)
$$

Among them, $W_{t}$ is the total threat; $W_{x}$ is the performance index price; $k_{w}$ is weight ratio of threat cost and performance constraints cost; $a$ is balance coefficient, which is used to adjust the order of magnitude of the threat cost and the constraint cost. Here researchers elect voyage cost as performance index price to restrain, quality of performance index includes two aspects, namely on the premise that route length should not exceed maximum range and threat cost is as low as possible.

\section{2) Route planning objective function:}

Modeling the impact of mission decision-making, the expectations of route planning objective function and the commander is consistent, namely a certain type of UAV can reconnoiter effectively at a minimum cost. So route planning objective function can be expressed as follows:

$$
F=\frac{V}{W}
$$

$V$ is the effectiveness of reconnoitering target, $W$ is the total cost of the route. According to model formula, the objective function can be transferred to:

$$
\max F=k_{\text {juece }} \frac{\sum_{i=1}^{N} L_{\text {effective }}}{k_{w} a W_{t}+\left(1-k_{w}\right) W_{x}}
$$

\section{3) The rules of ant state transition:}

The probability of an artificial ant that selects a new feasible node is determined by the price of the edge between two nodes and the intensity of the biological pheromone, the probability of ant $\mathrm{k}$ transform from the current node $r$ to feasible node $s$ is as follows:

$$
p_{k}(r, s)=\left\{\begin{array}{cc}
\frac{\tau(r, s)^{\alpha} \eta(r, s)^{\beta}}{\sum_{s \in J_{k}(r)} \tau(r, s)^{\alpha} \eta(r, s)^{\beta}} & \left(s \in J_{k}(r)\right) \\
0 & \text { otherwise }
\end{array}\right.
$$

In the above formula, $\tau(r, s)$ is the intensity of biological pheromone that the ant stored in the edge $V(r, s)$; $\eta(r, s)$ is the visibility of the node $s$ relative to the node $r$, $\eta(r, s)=1 / \mathrm{C}_{\mathrm{rs}}, C_{r s}$ is the cost of edge $V(r, s) ; J_{k}(r)$ is the set of ant $k$ that can reach all possible nodes from node $r$, these nodes are all adjacent nodes of node $r$, and they are more closer to the target than node $r ; \alpha, \beta$ are the control parameter, to determine the relative importance of the biological pheromone and visibility. The probability of all feasible selected nodes that ants transferred from state $r$ to state $s$ will increase with the strength of the biological pheromone, and reduce with the increase of channel cost.

\section{4) Rules of modifying biological pheromone:}

Once all the ants have completed their selection process of candidate path (to find a feasible solution of the path planning problem), they must comprehensively correct the pheromone on the edge. Correction rules are as follows:

$$
\tau(r, s)=(1-\rho) \cdot \tau(r, s)+\rho \cdot\left[\Delta \tau(r, s)+e \cdot \Delta \tau^{e}(r, s)\right]
$$

In this formula

$$
\Delta \tau(r, s)=\sum_{k=1}^{m} \Delta \tau^{k}(r, s)
$$

\section{5) The pheromone intensity:}

In order to guarantee that the ants can reach the target point, put a biological pheromone source in target point, attract ants move to target point. Assume that biological pheromones that spread the target points are weakened with the increase of distance in a reciprocal relationship, researchers can define the influence of biological pheromone spread by the target points on channel $V(r, s)$ as follows:

$$
\Delta \tau^{m}(r, s)=\left\{\begin{array}{cc}
A\left(1 / R_{s}-1 / R_{r}\right) / l_{r s} & \left(\mathrm{R}_{\mathrm{s}}\right)<R_{r} \\
0 & \left(\mathrm{R}_{\mathrm{s}}\right) \geq R_{r}
\end{array}\right\}
$$

In this formula, $A$ is the intensity of biological pheromone of the target point; $R_{s}$ is the distance of node $s$ from the target; $R_{r}$ is the distance of node $r$ from the target; $l_{r s}$ is the length of channel $V(r, s)$.

\section{6) Stimulating factor:}

Stimulating factor $\eta_{i j}=1 / d_{i j}$ is related to the distance, that is, the nearer the target is, the more stimulating for the ants.

\section{Implementation steps}

Specific steps of the algorithm are as shown in the chart of Fig. 3: ${ }^{10-12]}$

(1) Initialize subsidiary population that size is $P$, number is $n$;

(2) Evaluate all individual route;

(3) If meet the termination conditions turn to step (8), or turn to step (4);

(4) To choose $s$ of individuals from each population to form reproduction pool; 


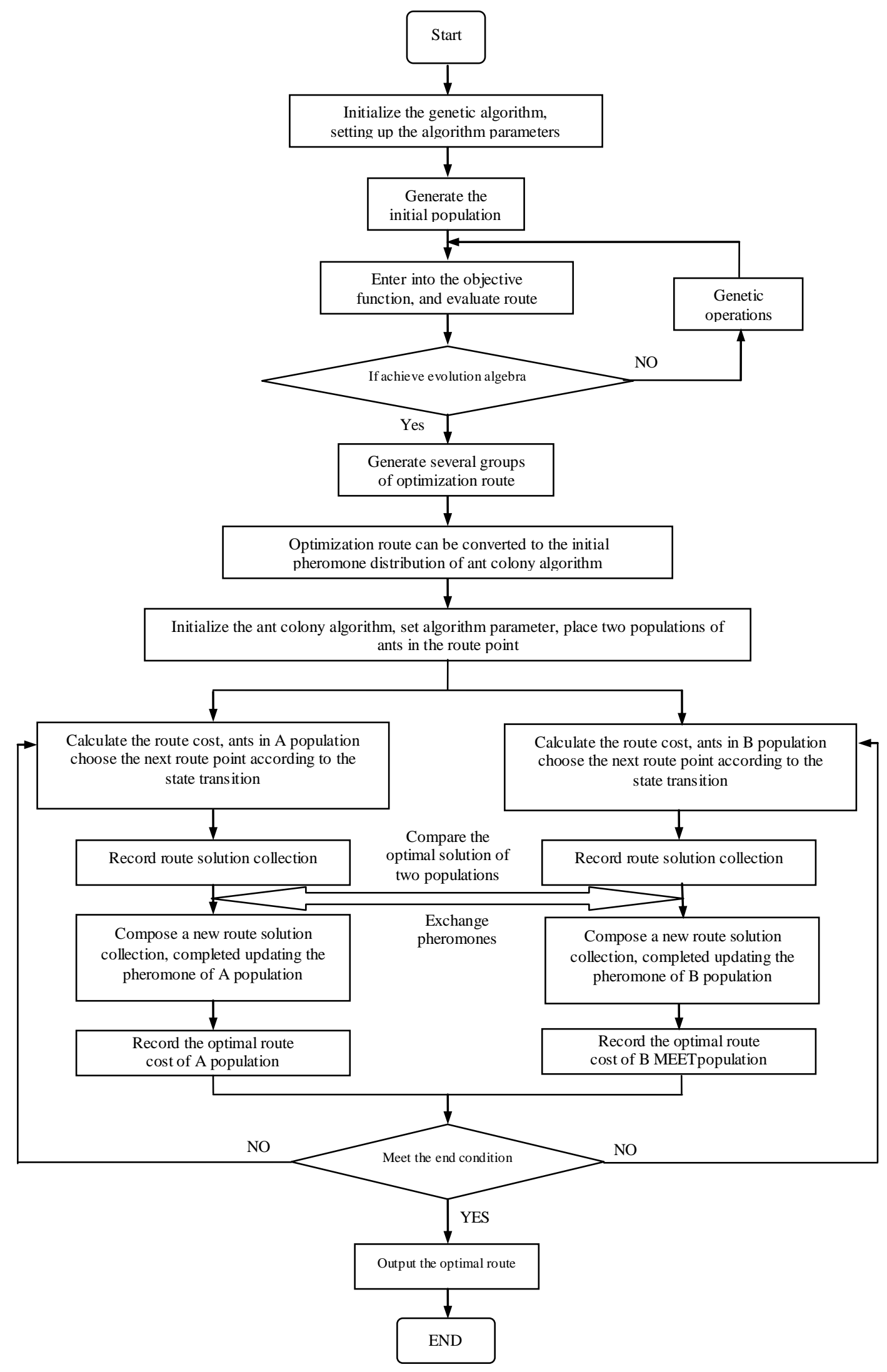

Figure 3. The flow chart of a type UAV route planning based on dual-population genetic ant colony algorithm 
(5) To select evolutionary factor, and operate the selected individual, then to generate $s$ new individuals;

(6) To evaluate new individual and insert it into his father's subsidiary population according to the category and individual price;

(7) To remove the final $s$ individuals in subsidiary population, restore the subsidiary population size, and return to step (3)

(8) Output the optimal route of each subsidiary population as the planning results;

(9) By using the genetic operation results, initialize the two population's parameters: $\alpha_{A}, \beta_{A}, \rho_{A}, Q_{A}, m_{A}, \alpha_{B}, \beta_{B}$, $\rho_{B}, Q_{B}, m_{B}, \lambda, \gamma$, and $\rho_{A} \in(0,1)$ get the minimum, and $\rho_{B} \in(0,1)$ get the maximum;

(10) For $N C=1$ : $N C_{\max }$ enter following cycle:

(11) Place the two populations $m_{A}$ and $m_{B}$ ants on the start node of route planning, calculate $\omega_{f}, \omega_{t}$ of adjacent feasible route node and the comprehensive cost $F$ according to the performance index;

(12) Two populations respectively calculate selective probability according to the rules of state transition, and then select the next node to reach and complete their travel;

(13) Two populations respectively update the pheromone on the path according to the rules of global updating rule and pheromone updating formula;

(14)Respectively record the best route of the two populations of $\mathrm{A}$ and $\mathrm{B}$ in the iteration;

(15) Compare the iterative optimal route of the two populations; take the shorter ones as the best route of this iterative result;

(16) When algorithm falling into local optimum, enter the population communication function, exchange the pheromone on corresponding trails of population A and B;

(17) When algorithm falling into local optimum, dynamically change the numerical value of $\rho_{A}$ and $\rho_{B}$ according to the bidirectional self-adaptive adjustment formula;

(18) $N C=N C+1$;

(19) End the cycle;

(20) Output the optimal route.

\section{THE EXPERIMENTAL SIMULATION}

Task description: the depth of enemy defense area is $200 \mathrm{~km}$, recently 4 new firepower deployment points in rear defense area appear, the UAV detachment is ordered to reconnoiter the situation. Known launch point $(A)$, target point $(\boldsymbol{\Delta})$ and threat point $(\bullet)$ coordinates are shown in Fig. 2 and table 1. ${ }^{[13][14]}$

Here researchers respectively assume the probability of mountains, radar, missiles, flack and thunderstorm destroying the UAV as $1,0.85,0.7,0.5$ and 0.1 , so the threat cost of UAV executing mission is as follows:

$$
W_{t}=1 \cdot P_{S f}+0.85 \cdot P_{R d}+0.7 \cdot P_{M d}+0.5 \cdot P_{M g}+0.1 \cdot P_{W e}
$$

Assuming the parameters in the simulation as follows: the parameters in the basic ant colony algorithm is set to $m=80, \alpha=2, \beta=5, \rho=0.95, Q=2, N C_{m a x}=100$; the parameters of genetic algorithm in dual population genetic ant colony algorithm are as follows, population size $M=50, P_{c}=0.6$, $P_{m}=0.2$; the parameters of ant colony algorithm are as follows, dual population size $m_{\mathrm{A}}=80, m_{\mathrm{B}}=80, \rho_{\mathrm{A}}=0.05$, $\rho_{\mathrm{B}}=0.95, \tau_{\min }=0.01, \tau_{\max }=4, \alpha=1, \beta=10, Q=1, N C_{\max }=100$.

TABLE I. THE COORDINATE AND RADIUS OF THE LAUNCH POINT ,TARGET AND THREAT POINT

\begin{tabular}{|c|c|c|c|}
\hline $\begin{array}{l}\text { launch point } \\
\text { coordinate }\end{array}$ & \multicolumn{3}{|c|}{$(0,50)$} \\
\hline $\begin{array}{c}\text { target } \\
\text { coordinate }\end{array}$ & \multicolumn{3}{|c|}{$(100,120),(125,108),(165,90),(115,30)$} \\
\hline \multirow{12}{*}{$\begin{array}{l}\text { threat point } \\
\text { coordinate }\end{array}$} & $\begin{array}{c}\text { The number and } \\
\text { type } \\
\text { of threat source }\end{array}$ & $\begin{array}{c}\text { coordinate } \\
(\mathrm{km})\end{array}$ & $\operatorname{radius}(\mathrm{km})$ \\
\hline & 1 Ordinary radar & $(70,60)$ & 15 \\
\hline & 2 Ordinary radar & $(120,140)$ & 16 \\
\hline & 3 Ordinary radar & $(180,60)$ & 15 \\
\hline & $\begin{array}{l}4 \text { Antiaircraft } \\
\text { missile }\end{array}$ & $(32,60)$ & 12 \\
\hline & $\begin{array}{l}5 \text { Antiaircraft } \\
\text { missile }\end{array}$ & $(140,150)$ & 18 \\
\hline & $\begin{array}{c}6 \text { Antiaircraft } \\
\text { artillery }\end{array}$ & $(40,80)$ & 15 \\
\hline & $\begin{array}{c}\text { 7Antiaircraft } \\
\text { artillery }\end{array}$ & $(60,88)$ & 20 \\
\hline & 8 mountain & $(56,20)$ & 11 \\
\hline & 9 mountain & $(90,135)$ & 10 \\
\hline & 10 thunderstorm & $(130,70)$ & 22 \\
\hline & 11 thunderstorm & $(40,150)$ & 30 \\
\hline
\end{tabular}

\section{A. Circumstance 1}

Commander orders that UAV cannot traverse the threatened area, but can finish the photographic reconnaissance with a format camera at the same time. Therefore, set the threat avoidance coefficient $\delta=1$ and the efficient target reconnaissance range $d \leq 3.6$.

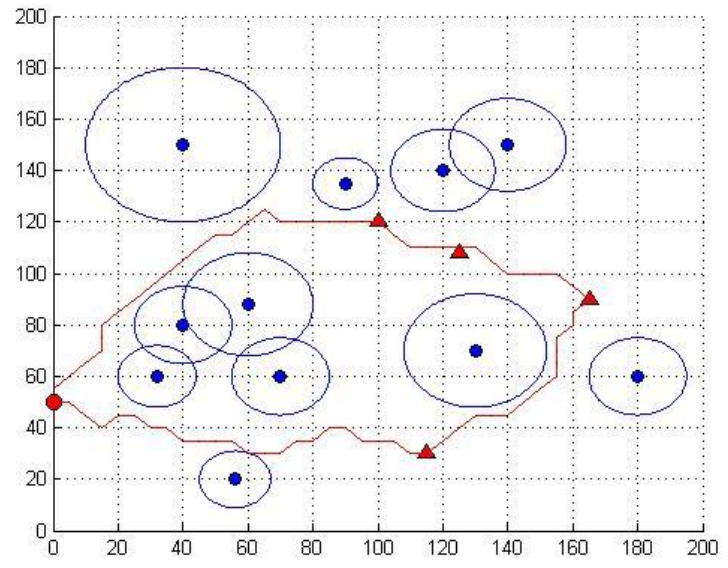

Figure 4. the UAV optimal route 1 
The UAV route length is $441.6295 \mathrm{~km}$. Obtained from the simulation results, the flight route can avoid threat area and can effectively detect the sky above target, the route is in accordance with the operational needs of a certain UAV.

\section{B. Circumstance 2}

Commander orders that UAV can appropriately traverse the threatened area and finish the photographic reconnaissance with format camera at the same time. Therefore, set the threat avoidance coefficient $\delta=0.85$, the efficient target reconnaissance range $d \leq 4$.

The UAV route length is $432.8427 \mathrm{~km}$, about $7.79 \%$ of which is in the threatened area. Obtained from the simulation results, the flight route can avoid most of the threat area, and can effectively detect the sky over the target, the route is in accordance with the operational needs of a certain UAV.

In order to validate the advantages of dual population genetic ant colony algorithm, researchers also use basic ant colony algorithm for route optimization with more than eighty percent of the results presenting stagnation phenomenon as a result, the rest of the results can't do better than the results of the improved algorithm.

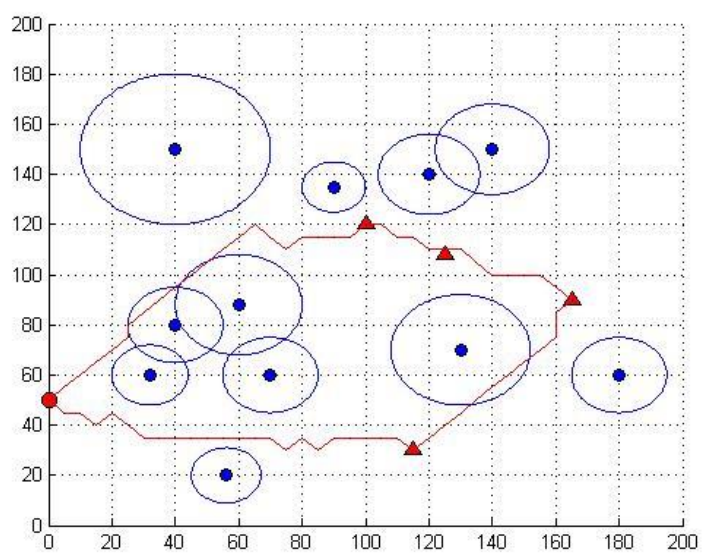

Figure 5. the UAV optimal route 2

\section{CONCLUSION}

The study of UAV route planning is of great military value and important realistic significance. It can provide powerful guarantee for UAV obtaining effective battlefield intelligence. Researchers establish the route planning model of a certain type UAV which reflects the characteristics of the techniques and tactics of UAV and the battlefield environment when UAV executes tasks. Dual population genetic ant colony algorithm is put forward which is better than the basic ant colony algorithm in search performance and precise solution. Route planning of a certain type of UAV is designed on the basis of improved ant colony algorithm. The feasibility and effectiveness of the scheme is proved by simulation experiments.

Of course, further research is needed for this paper, such as how to anew plan route when UAV is confronted with unexpected threat, and how to extend twodimensional route planning put forward in the paper to three-dimensional route planning. These are all of great value for research.

\section{ACKNOWLEDGMENT}

This work was supported by National Natural Science Foundation of China (No.41374156) and Anhui Provincial Natural Science Foundation (No.1308085MD54, No.1508085MF108).

\section{REFERENCES}

[1] Dorigo, M. Ant Colony Optimization[M]. Beijing: Tsinghua University press, 2007.1

[2] Min changwan. Introduction of Military Aircraft Route Planning[J]. Flight dynamics, 1998,16(4) 14-19

[3] $\mathrm{Hu}$ zhonghua. Unmanned Aerial Vehicle Route Planning Technology Research and Development Ttrend [J] Avionics technology.2009.6

[4] Zhang Zhixie. Solving Optimal Path Problem Based on Improved Ant Colony Algorithm[J]. Computer System Applications, 2012,21(10):76-80

[5] Maliang. Ant Colony Optimization Algorithm[M]. Beijing: Science Press, 2008.2

[6] Yao Yongjie. Path Planning for UAVs Based on Improved GA Algorithm[J]. Computer Simulation,2011,28(6):44-48

[7] Han Pan. Path Planning for UAVs Based on Improved Ant Colony Algorithm[J]. Journal of Jilin Uuiversity(Information Science Edition),2013,31(1):66-72

[8] Zhu Kaiyan. Dual Population Ant Colony Optimization Based on Pheromone Diffusion[J]. Microcomputer Applications, 2010, 31(7): 22-26

[9] Duan Haibin. Ant Colony Algorithm Theory and Applications[M]. Beijing: Science Press, 2005.12

[10] Wang Xuzhi, etc. Path Planning for UAV Based on Ant Colony Algorithm and Dynamic Simulation[J]. Command Control \& Simulation, 2012,34(1):29-32

[11] Xiong Yu. Route Planning of UAV Based on Improved Ant Colony Algorithm[J]. Computer \& Digital Engineering, 2010, 38(7): 41-45

[12] Liu senqi. Unmanned Combat Aircraft Route Planning of based on Voronoi Diagram and the Ant Colony Optimization Algorithm[J].Journal of system simulation,2008.11

[13] Li Shiyong. Ant Colony Algorithms with Applications[M] Harbin Harbin Institute of Technology press, 2004.9

[14] Rao yuedong. Application Research of Route Planning of UAV based on Improved Ant Colony Algorithm[D]. Wuhan. Wuhan University of Technology, 2010 